\title{
TARMED Suisse
}

\section{Ernst Gähler ${ }^{a}$, Irène Marty ${ }^{b}$}

a Dr, Vice-président de la FMH, Responsable du domaine Tarifs et conventions

b Cheffe du service Tarifs et conventions

\section{Projet 2010}

La révision urgente de la structure tarifaire du TARMED est actuellement bloquée en raison de points de vue divergents quant à une convention sur la régulation des coûts et leurs effets. Les propositions faites par santésuisse concernant les points tarifaires sont inacceptables pour le corps médical. Néanmoins, quelques ébauches de solutions ont maintenant été trouvées dans un entretien au sommet mené récemment entre santésuisse et la FMH: la régulation des coûts pourra avoir lieu par le biais d'une LeiKoV modifiée, dont l'application sera contraignante pour les sociétés cantonales de médecine.

\section{Révision du concept de la valeur intrinsèque} La réglementation transitoire relative au projet Valeurs intrinsèques/prestations de droits acquis est publiée sur le site internet de TARMED Suisse (www.tarmed suisse.ch/184.html). Dès le $1^{\text {er }}$ janvier 2010, les positions de droits acquis seront réglées sur la base de diplômes de formation continue.

\section{Indemnisation de la vaccination H1N1 pour le cabinet du médecin de famille (vaccination ambulatoire)}

Comme la vaccination H1N1 n'aura pas lieu sous forme de vaccination de masse, la FMH et la CCM ont exigé que le corps médical participe aux négociations sur un forfait car on aimerait éviter un scénario sem-

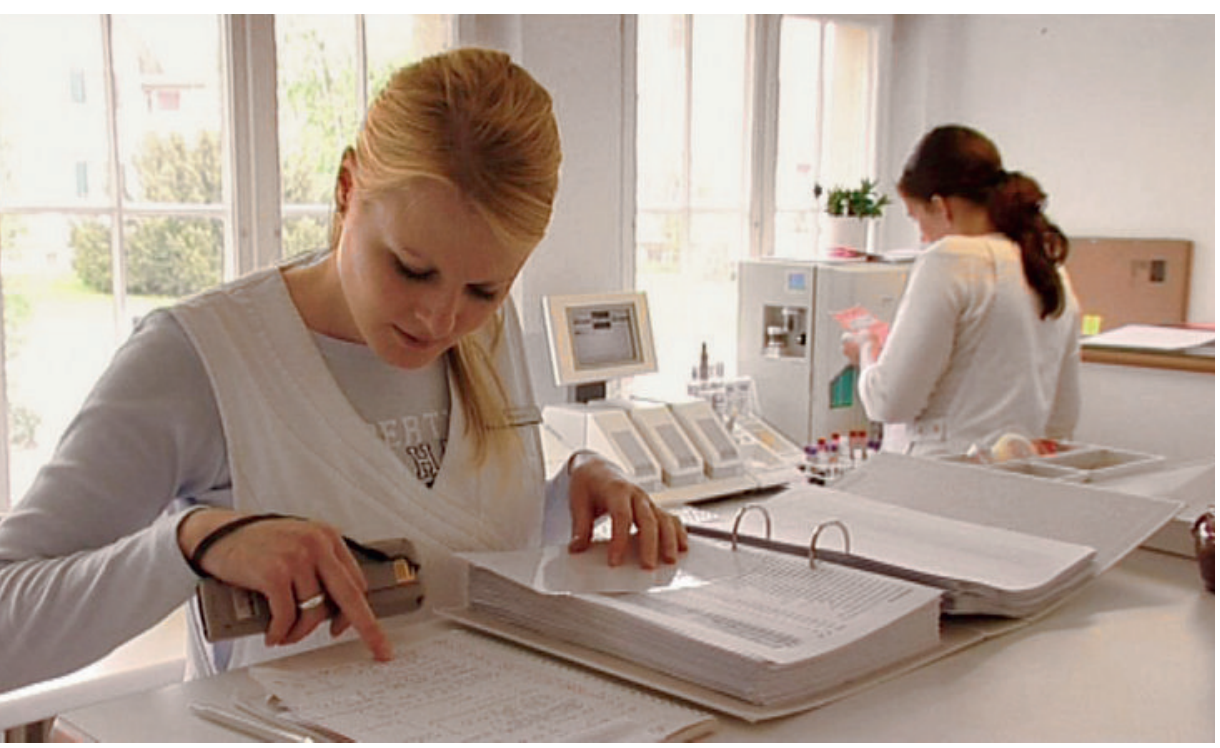

blable à celui de la vaccination HPV. Lors d'âpres discussions, on a convenu d'un forfait de Fr. 19.50 par injection, le vaccin étant gratuit. Après avoir consulté les sociétés cantonales de médecine et les sociétés de discipline médicale concernées, la FMH a envoyé à l'OFSP une «Letter of Intent» lui donnant son accord. En revanche, un forfait de Fr. 17.15 a été prévu pour la vaccination de masse en cas de pandémie.

\section{Réduction de la marge de distribution pour la propharmacie}

Le conseiller fédéral Pascal Couchepin a décidé d'abaisser la marge de distribution de $15 \%$ à $12 \%$ au $1^{\text {er }}$ octobre 2009. Les corrections de prix entreront en vigueur le $1^{\text {er }}$ mars 2010; les anciennes marges seront ainsi valables jusqu'au 28 février 2010. Les médecins disposant d'une pharmacie au cabinet médical devront donc adapter leurs stocks à cette date.

\section{Révision de la Liste des analyses}

Répercussions de la révision de la Liste des analyses - premiers résultats

Nos mesures confirment (malheureusement) pour la troisième fois de suite (juillet, août et septembre) les craintes et les simulations que nous avions déjà publiées avant l'introduction de la nouvelle Liste:

- Pour les médecins de premier recours (généralistes / internistes), nous mesurons une perte de $25 \%$ sur le chiffre d'affaires du laboratoire.

- Par contre, une société de discipline «gagnante» verra son chiffre d'affaires augmenter de $30 \%$

- On aperçoit déjà maintenant des tentatives de déplacement de prestations.

Afin d'effectuer les mesures pertinentes suffisamment tôt, nous avons élaboré une méthode spéciale et l'avons rendue plausible au moyen de valeurs enregistrées dans le passé. Mais pour pouvoir clarifier de manière complète les effets tout au long de la chaîne des processus, il nous faut disposer des données des laboratoires industriels.

\section{Monitorage INFRAS/OFSP}

Le concept a été adopté et l'entreprise INFRAS a été chargée de le mettre en œuvre. Contrairement aux instructions de la CdG-N, l'OFSP a refusé d'examiner également dans le monitorage la facturation effectuée 
selon des critères objectifs et propres à l'économie d'entreprise. La FMH va conclure une convention avec INFRAS pour la livraison et la gestion de ses données, l'accompagnement du projet et la prise d'influence.

\section{Monitorage élargi de la FMH}

Le Comité central et l'Assemblée des délégués ont décidé d'effectuer un monitorage élargi et d'examiner toute la chaîne des analyses, y compris les laboratoires industriels. L'objectif visé de ce monitorage est de suivre les répercussions sur la LeiKoV ainsi que la facturation des analyses selon des critères objectifs et propres à l'économie d'entreprise. Pour pouvoir effec- tuer un monitorage complet, nous avons aussi besoin des données des laboratoires auxquels vous confiez des analyses. A cet effet, nous avons déjà publié dans notre dernière «Info tarifaire importante» (Bulletin des médecins suisses 36/2009) un coupon par lequel vous nous permettez d'obtenir les données des laboratoires industriels. Nous avons déjà reçu plus de 500 coupons à ce jour et en remercions cordialement tous les expéditeurs-trices! Nous serions heureux si tous les médecins disposant d'un laboratoire au cabinet médical pouvaient renvoyer ce coupon à: FMH, Tarifs et conventions, Gösgerstrasse 8, 4600 Olten. Nous le publions encore une fois:

\section{Coupon de la FMH pour le monitorage des laboratoires}

Par l'envoi de ce coupon, je charge mon/mes laboratoire(s) d'analyses d'envoyer une copie de mes mandats de laboratoire au pool de données. Les éventuelles données des patients doivent être traitées anonymement; le pool de données sert exclusivement au monitorage de la Liste des analyses. Ce mandat n'entraîne aucun coût pour moi.
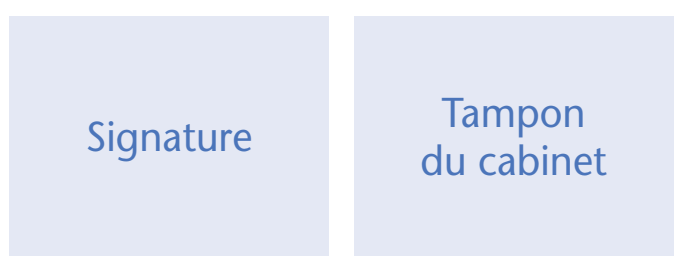

\section{№ EAN}

Veuillez renvoyer ce coupon dans les plus brefs délais à: FMH, Tarifs et conventions, Gösgerstrasse 8, 4600 Olten



Mes laboratoires privés/industriels 